\title{
Reasoning in knowledge bases with extrenal and internal uncertainties
}

\author{
Phan Dinh Dieu \& Tran Dinh Que \\ Institute of Information Technology
}

\section{Introduction}

In the recent years, in A.I. a great deal of at tention has been devoted to formalisms dealing with various aspects of reasoning with uncertain information, and a number of theories and methods for handling uncertainty of knowledge has been proposed, notably, the probability theory, the Dempster-Shafer theory, and the possibility theory. Among these theories, the propobalify one is surely the most developed by its long history and elaborated foundations.

In this paper the uncertainty of a sentence will be given by an interval of possible values for its truth probability. Two types of knowledge with uncertainty will be investigated: external uncertainty and internal uncertainty The former is given in the form $\langle S, I\rangle$, in which $S$ is a sentence, and $I=[\mathrm{a}, \mathrm{b}]$ is a closed subinterval of the unit interval $[0,1]$; it means that the truth probability of the whole sentence $S$ lies in the interval $I . I$ is then called the interval of truth probabities of $S$. In the later case, intervals of truth probabilities are given to subsentences of the given sentence $S$. For example,

$$
<S_{1}, I_{1}>\wedge<S_{2}, I_{2}>\wedge_{i:} \cdots \wedge<S_{n}, I_{n}>\rightarrow \leqslant S_{n+1}, I_{n+1}>
$$

is a knowledge with internal uncertainty.

Let $\mathcal{B}$ be a knowledge base with these types of uncertainty and $S$ be a any sentence. A semantics, which underlies a method of deducing the interval of truth probabilities of $S$ from $\mathcal{B}$, will be given. 
The paper is structurcd as follows: In Section 2 we shall review the probabilistic logic by N.J. Nilsson, and its extention to probabilistic logic with interval values. In Section 3, we shall discuss a method of reasoning from a set of knowledges with internal uncertainty. Section 4 is devoted to a method of reasoning from a knowledge base containing both external and internal uncertainties. Some illustrative examples will be given in Section 5 .

\section{External Uncertainty}

We shall use methods of the interval-valued probabilistic logic for reasoning in knowledge bases with external uncertainty. This logic is based on the semantics given by N.J. Nilsson [4], and is presented in details, e.g., in [6].

Let us give a knowledge base

$$
\left.\mathcal{B}=\left\{<S_{i}, I_{i}{ }^{i}\right\rangle \mid i=1, \ldots, L\right\},
$$

and let $S$ be a target sentence. We put $\sum=\left\{S_{1}, \ldots S_{L}, S\right\}$, and suppose that $W_{1}, \ldots, W_{k}$ are all $\sum$ - classes of possible worlds. Every class $W_{j}$ is characterized by a consistent vector $\left(\boldsymbol{u}_{1 j}, \ldots, u_{\varepsilon_{j}}, u_{j}\right)$ of truth values of sentences $S_{1}, \ldots S_{L}, S$.

Given a probability distribution $\left(p_{1,}, \ldots p_{K}\right)$ over the classes. $W_{1}, \ldots, W_{K}$, the truth probability $y_{1 i}$ of sentence $S_{i}$ is defined to be the sum of probabilities of classes of worlds in which $S_{i}$ is true, i.e., $\pi\left(S_{i}\right)=u_{i 1} p_{1}+\cdots+u_{i K} p_{K}$. From this semantics it follows that, given the knowledge base $\mathcal{B}$, the derived interval-value $[\alpha, \beta]$ for the truth probability of the sentence $S$ is defined by

$$
\alpha=\min \pi(S), \quad \beta=\max \pi(S),
$$

where

$$
\pi\left(S_{i}\right)^{\prime}=u_{1}^{\prime} p_{1}+\cdots+u_{K} p_{K}
$$

subject to the constraints

$$
\left\{\begin{array}{l}
\pi_{i}=u_{i 1} p_{1}+\cdots+u_{i K} p_{K} \in I_{i}(i=1, \ldots, L) \\
\sum_{j=1}^{K} p_{j}=1, p_{j} \geq 0(j=1 \ldots, K) .
\end{array}\right.
$$

We denote the interval $[\alpha, \beta]$ by $F(S, \mathcal{B})$, and write $\mathcal{B} \vdash<S, F(S, \mathcal{B})>$.

Now, let us give a set of sentence $\Gamma$. We define $\mathcal{I}$ to be the set of all mappings from $\Gamma$ into $C[0,1]$ - the set of closed subintervals of $[0,1]$. A such mapping $I$ assigns to each sentence $P \in \mathrm{\Gamma}$ an interval $I(P) \in C[0,1]$.

The given knowledge base $\mathcal{B}$ defines an operator $R_{\mathcal{B}}$ from $\mathcal{I}$ into $\mathcal{I}$ as follows: For every $I \in \mathcal{I}$, we establish a new knowledge base

$$
\mathcal{B}^{\prime}=\mathcal{B} \cup\{<P: H(P)>\mid P \in \Gamma\}
$$


and then we take for every $P \in \Gamma$ the interval $I^{\prime}(P)=F\left(P, \mathcal{B}^{\prime}\right)$. The mapping $I^{\prime}$ is $R_{B}(I)$ defined to be the image of $I$ by the operator $R_{\mathcal{B}}: R_{\mathcal{B}}(I)=I^{\prime}$.

It is easy to see that if $R_{\mathcal{B}}(I)=I^{\prime}$ then $R_{\mathcal{B}}\left(I^{\prime}\right)=I^{\prime}$; therefore

$$
R_{\mathcal{B}}^{n}(I)=R_{\mathcal{B}}(I) \text { for any } n \geq 1
$$

The calculation of the operator $R_{\mathcal{B}}$ is reduced to the solution of linear programming problems which has to face up a very large computational complexity whenever the sizes of $\mathcal{B}$ and $\Gamma$ are large. Some at tempts to reducing the size of linear programming problems have been investigated, e.g., a method of reduction is given in [7] for the cases when the core $\left\{S_{1}, \ldots, S_{L}\right\}$ of $\mathcal{B}$ forms a logic program.

Instead of the method presented above we can use methods of approximate reasoning, e.g., by means of deductions based on inference rules (see [2]), however, in this case the property $\left({ }^{*}\right)$ may not be satisfied.

\section{Internal Uncertainty}

In this section a method of reasoning on knowledges with internal uncertainty will be discussed. We limit ourself to consider knowledges given by rules of the form:

$$
<S_{1}, I_{1}>\wedge \cdots \wedge<S_{n}, I_{n}>\rightarrow<S, I>
$$

Let us given a knowledge base $\mathcal{B}=\left\{J_{j} \mid j=1, \ldots, M\right\}$, where $J_{j}$ is the rule:

$$
J_{j}=\left\langle A_{j 1}, I_{j 1}>\wedge \cdots \wedge<A_{j m}, I_{j m_{j}}>\rightarrow<A_{c_{j}}, I_{c_{j}}>.\right.
$$

Let $\Gamma$ be a set of sentences containing all sentences occuring in rules $J_{j}(j=1 \ldots M)$ of $\mathcal{B}$. As above we denote by $\mathcal{I}$ the set of mappnings $I$ from $\Gamma$ to $C[0,1]$.

For any $I_{1}, I_{2} \in \mathcal{I}$, we say that $I_{1} \leq I_{2}$ iff $I_{1}(P) \subseteq I_{2}(P)$ for every $P \in \Gamma$.

We say that the rule $J_{j}$ is satisfied by the mappning $I \in \mathcal{I}$, iff $I\left(A_{j k}\right) \subseteq I_{j_{k}}$ for every $k=1, \ldots, m_{j}$. Note that if $m_{j}=0$ then $J_{j}$ is satisfied by any mapping $I$.

Now we define an operator $t_{B}$ from $I$ into $\mathcal{I}$, which transforms any $I \in \mathcal{I}$ into a mapping $t_{\mathcal{B}}(I)$ such that for every $P \in \Gamma$ :

$$
t_{\mathcal{B}}(I)(P)=I(P) \cap\left(\bigcap_{j \in E} I_{c_{j}}\right)
$$

where $E=\left\{j \mid A_{c_{j}}=P\right.$ and $J_{j}$ is satisfied by $\left.I\right\}$. Here. for the $\cdots 1$ assume that $\bigcap_{j \in E} I_{c_{j}}=[0,1]$ whenever $E=\emptyset$.

We have the following proposition: 
Proposition 1. For any mapping $I \in \mathcal{I}$ there exists always a natural number $n$ such that $t_{\mathcal{B}}^{n+1}(I)=t_{\mathcal{B}}^{n}(I)$. In other orlds, the process of iteration of the oprrator $t_{\mathcal{B}}$ on any given $I \in \mathcal{I}$ always halts after a finite number of steps.

Proof. Suppose that $E_{0}, E_{1}, E_{2}, \ldots$ are the set of indexes of rules which are satisfied satisfied by $I, t_{\mathcal{B}}(I), t_{\mathcal{B}}^{2}(I), \ldots$, respectively.

Let $h_{i}=\left|E_{i}\right|(i=1,2, \ldots)$ be the number of elements of the set $E_{i}\left\{h_{i} / i=1,2, \ldots\right\}$ is then a sequence of integers such that

$$
0 \leq h_{0} \leq h_{1}, \ldots \leq h_{n} \leq \ldots \leq M
$$

Consider two cases

(i) There exists a number $n$ such that $h_{n-1}=M$, i.e., $J_{j}$ is satisfied by the mapping $t_{\mathcal{B}}^{n-1}(I)$ for every $J=1, \ldots, M$. In this case we have $t_{\mathcal{B}}^{n}(I)=t_{\mathcal{B}}^{n+1}(I)$.

(ii) There exists a number $n$ such that $h_{n-1}=h_{n}<M$. In this case we have $E_{n-1}=$ $E_{n}$, and it is easy to see that

$$
t_{\mathcal{B}}^{n}(I)=t_{\mathcal{B}}^{n+1}(I)
$$

The proposition has been proved.

From this proposition we can define an operator $T_{\mathcal{B}}$ as follows: For any $I \in$ $\mathcal{I}, T_{\mathcal{B}}(I)=t_{\mathcal{B}}^{n}(I)$, where $n$ is the least number such that $t_{\mathfrak{B}}^{n}(I)=t_{\mathfrak{B}}^{n+1}(I)$.

Let $S$ be a sentence. We denote by $\Gamma$ the set consisting of $S$ and of all sentences occuring in rules $J_{j}$ of $\mathcal{B}$. Let $I$ be the mapping which assigns the interval $[0,1]$ for every sentence in $\Gamma$. Then $T_{\mathcal{B}}(I)(S)$ can be considered as the interval-value for the truth probability of the sentence $S$ clerived from the knowledge base $\mathcal{B}$.

\section{A Method of Reasoning.}

We consider now the knowledge bases consisting both types of knowledges with external and internal uncertainty. Let $\mathcal{B}$ be such a knowledge base, we can write $\mathcal{B}=\mathcal{B}^{E} \cup \mathcal{B}^{I}$, where $\mathcal{B}^{E}$ consists of knowledges with external uncertainty. Suppose that

$$
\begin{gathered}
\mathcal{B}^{E}=\left\{<S_{i}, I_{i}>\mid i=1, \ldots, L\right\}, \\
\mathcal{B}^{I}=\left\{J_{j} \mid j=1, \ldots, M\right\},
\end{gathered}
$$

where

$$
J_{j}=<A_{j 1}, I_{j 1}>\wedge \cdots \wedge<A_{j m_{j}}, I_{j m_{j}}>\rightarrow<A_{c_{j}}, I_{c_{j}}>.
$$

Let $S$ be any (target) sentence. Our problem is to deduce from the knowledge base $\mathcal{B}$ the interval value for the truth probability of the sentence $S$. 
For this purpose we put $\Gamma$ to be the set consisting of the sentence $S$ and all distinct sentences occuring in $\mathcal{B}^{E}$ and $\mathcal{B}^{I}$. Denote by $\mathcal{I}$ the set of mappings from $\Gamma$ to $C[0,1]$. Let $I_{0}$ be the mapping defined by

$$
I_{0}(P)= \begin{cases}I_{i}, & \text { if } P=S_{i} \text { for some } i=1, \ldots, L \\ {[0,1],} & \text { otherwise. }\end{cases}
$$

$I_{0}$ is called the initial assignment (of interval-value to sentences in $\Gamma$ ).

Now we define a sequence of assignments $I_{n}(n=0,1, \ldots$,$) initiated by I_{0}$ and given recursively as follows:

$$
I_{n}= \begin{cases}\mathcal{R}\left(I_{n-1}\right), & \text { if } n \text { is odd } \\ \mathcal{T}\left(I_{n-1}\right), & \text { if } n \text { is positive even. }\end{cases}
$$

Here $\mathcal{R}$ stands for $R_{\mathcal{B}^{E}}$, and $\mathcal{T}$ stands for $T_{\mathcal{B}^{I}}$.

Proposition 2. Let $\mathcal{B}$ be a knowledge base, and $S$ be any given sentence. There exists a natural number $n$ such that $I_{n+2}=I_{n+1}=I_{n}$.

Proof. From the definition of the sequence $\left\{I_{n}\right\}(i=0,1, \ldots)$ we can write

$$
I_{0} \stackrel{\mathcal{R}}{\mapsto} I_{1} \stackrel{\mathcal{T}}{\mapsto} I_{2} \stackrel{\mathcal{R}}{\mapsto} \ldots \stackrel{\mathcal{R}}{\mapsto} I_{n-2} \stackrel{\mathcal{T}}{\mapsto} I_{n-1} \stackrel{\mathcal{R}}{\mapsto} I_{n} \stackrel{\mathcal{T}}{\mapsto} I_{n+1} \stackrel{\mathcal{R}}{\mapsto} \ldots
$$

Let $h_{i}$ be a number of rules $J_{i}$ satisfied by $I_{i}(i=1,3,5, \ldots)$. Then, $\left\{h_{i}\right\}$ is a sequence of integers such that

$$
0 \leq h_{1} \leq h_{3} \leq \cdots \leq h_{n-2} \leq h_{n} \leq \cdots \leq M .
$$

Consider two cases

(i) There exists a number $n$ such that $h_{n-2}=M$, i.e, $J_{j}$ is satisfird by the mapping $I_{n-2}$, for every $j=1, \ldots, M$. Then, any rule $I_{j}(j=1, \ldots, M)$ is also satisfied by the mappings

$$
\begin{aligned}
I_{n-1} & =\mathcal{T}\left(I_{n-1}\right) \\
I_{n} & =\mathcal{R}\left(I_{n}\right)
\end{aligned}
$$

Thus

or $I_{n}=I_{n+1}=I_{n+2}$

$$
\begin{aligned}
& I_{n+1}=\mathcal{T}\left(I_{n}\right)=I_{n} \\
& I_{n+2}=\mathcal{R}\left(I_{n+1}\right)=\mathcal{R}\left(I_{n}\right)=I_{n}
\end{aligned}
$$

(ii) There exists a number $n$ such that $h_{n}=h_{n-2}<M$. Then the sets of rules satisfied by $I_{n}$ and by $I_{n-2}$ are the same. Therefore,

$$
\begin{aligned}
& I_{n+1}=\mathcal{T}\left(I_{n}\right)=I_{n} \\
& I_{n+2}=\mathcal{R}\left(I_{n+1}\right)=I_{n+1}
\end{aligned}
$$

and we have again $I_{n}=I_{n+1}=I_{n+2}$.

This completes our proof. 
Let $n$ be the least number having the property sated in the proposition 2 . We denote this $I_{n}$ by $I^{*}$, and call it to be the resulting assignment deduced from $\mathcal{B}$ to sentences in $\Gamma$.

The interval $I^{*}(S)$ is defined to be the interval value for the truth probability of the scntence $S$ derived from the knowledge base $\mathcal{B}$. We write also:

$$
\mathcal{B} \vdash<S, I^{*}(S)>\text {. }
$$

\section{Examples}

This section presents two examples illustrating the method of reasoning in a knowledge base consisting of both types of knowledge with internal and external uncertainty.

Example 1.

. : b::

Given a knowledge base $\mathcal{B}=\mathcal{B}^{E} \cup \mathcal{B}^{I}$ where $\mathcal{B}^{E}$ is the set of sentences

$$
\begin{aligned}
B \rightarrow A & :[1,1] \\
A \rightarrow C & :[1,1] \\
B & :[.2, .6] \\
C & :[.6, .7]
\end{aligned}
$$

and $\mathcal{B}^{I}$ is the set of rules

$$
\begin{array}{r}
J_{1}=C:[.5, .7] \longrightarrow B:[.3, .5] \\
J_{2}=B:[.2, .5] \wedge C:[.5, .7] \longrightarrow A:[.2, .5]
\end{array}
$$

Calculate the interval of truth probalilities of the sentence $A$.

Step 1. Applying the operator $\mathcal{R}$, we get

$$
\begin{aligned}
& A:[.2, .7] \\
& B:[.2, .6] \\
& C:[.6, .7] .
\end{aligned}
$$

Step 2. Both rules $J_{1}$ and $J_{2}$ are satisfied, so applying the operator $\mathcal{T}$ wo obtain

$$
\begin{aligned}
& A:[.2, .5] \\
& B:[.3,5] \\
& C:[.6, .7] .
\end{aligned}
$$

Step 3. Iterate $\mathcal{R}$, where $B:[.2, .6]$ is now replaced by $B:[.3, .5]$, we get

$$
A:[.3, .5] \text {. }
$$

As both $J_{1}$ and $J_{2}$ are satisfied after step 1 , it is not necessary to repeat $\mathcal{T}$ after step 3 and we get the finall result $A:[.3, .5]$.

Note that $\mathcal{B}^{E}$ is a type-A problem (see [2]); hence, we can apply the type-A rules to computing the intervals for $A, B, C$ instead of solving linear programming problems. 
Example 2. This example is more complex than the above; it illustrates the iteration of the operator $\mathcal{T}$. Suppose that we wish to derive the interval of truth probabilities of the sentence $A \wedge D$ from the knowledge base $\mathcal{B}=\mathcal{B}^{E} \cup \mathcal{B}^{I}$ where $\mathcal{B}^{E}$ is the set defined as follows

$$
\begin{aligned}
B \rightarrow & A:[.9,1] \\
D \rightarrow & B:[.8, .9] \\
A \rightarrow & C:[.6, .8] \\
& D:[.8,1] \\
& C:[.2,4]
\end{aligned}
$$

and $\mathcal{B}^{I}$ is the set of the rules $J_{j}(j=1,2,3)$ :

$$
\begin{array}{r}
J_{1}=C:[.1, .5] \longrightarrow B \wedge D:[.7,1] \\
J_{2}=C:[.2, .3] \wedge(B \wedge D):[.7, .9] \longrightarrow A \wedge D:[.4, .7] \\
J_{3}=A \wedge D:[.6, .9] \longrightarrow C:[.2, .3] .
\end{array}
$$

Step 1 Applying $\mathcal{R}$, we have

$$
\begin{aligned}
A & :[.2, .8] \\
B \wedge D & :[.6, .9] \\
A \wedge D & :[.5, .8] \\
C & :[.2, .4] \\
D & :[.8,1] .
\end{aligned}
$$

Step 2. Since only $J_{1}$ is satisfied, we get

$$
B \wedge D:[.7, .9]
$$

and the interval values of $A, A \wedge D, C, D$ are not varied.

Step 3. Repeating $\mathcal{R}$, only the interval value of $A \wedge D$ is varied

$$
A \wedge D:[.6,8]
$$

Step 4. Repeating $\mathcal{T}$, now $J_{3}$ and $J_{2}$ are satisfied, so we have

$$
\begin{array}{r}
C:[.2, .3] \\
A \wedge D:[.6, .7] .
\end{array}
$$

Step 5. $\mathcal{B}^{E}$ is now changed into $\mathcal{B}^{\prime}$ which consists of:

$$
\begin{array}{r}
B \rightarrow A:[.9,1] \\
D \rightarrow B:[.8, .9] \\
A \rightarrow C:[.6, .8] \\
D:[.8,1] \\
C:[.2, .3] .
\end{array}
$$


$\mathcal{R}$ is repeated and we have

$$
A \wedge D:[.6 . .7]
$$

By virtue of that all rules in $\mathcal{B}^{I}$ are satisfied in step 4 . the interval value for the truth probability of $A \wedge D$ is $[.6, .7]$.

\section{References}

1. Baldwin J.F., Evidential support logic programming, Fuzzy Sets and Systems. v $24(1987)$, p. 1-26.

2. Frisch A.M. \& Haddawy P., Anytime deduction for probabilistic logic, October 21, 1992 (Submitted for publication).

3. Genescreth M.R. \& Nilsson N.J., Logical foundations of Artificial Intelligencf Morgan Kaufman Publ. Inc. Los Altos, $\mathrm{CA}_{\mathrm{A}} \mathrm{A}, 1987$.

4. Nilsson N.J., Probabilistic logic, Artificial Intellig'nce 28(1986), p.71-78.

5. Phan I.D., Probabilistic logic for approximate reasoning, in I. Plander (ed.) Artificial Intelligence and Information Control System of Robots-89 (North Holland 1989), p. 107-112.

6. Phan D.D. On a theory of interral-valued probabilistic logic, Research Report, NCSR Vietnam, Hanoi 1991.

7. Phan D.D. \& Phan H.G., Interval-valued probabilistic logic for logic programs, March 1993 (to appear).

8. Raymond Ng. \& Subrahmanian V.S., Probabilistic logic programming, Information and Computation v. 101 (1992), p. 150-201.

\section{Abstract}

The paper presents a method of logical reasoning in knowledge bases with uncertainty; such a knowledge base is given by a set. of "knowledges" of two following forms:

(1) $\langle S, I\rangle$ where $S$ is a sentence, and $I \subseteq[0,1]$ is an interval of the possible values for truth probability of $S$.

(2) $\left\langle S_{1}, I_{1}>\wedge<S_{2}, I_{2}>\wedge \cdots \wedge<S_{n}, I_{n}>\rightarrow<S, I>\right.$, where $S_{1}, \ldots, S_{n}, S$ are sentences, and $I_{1}, \ldots, I_{n}, I$ are the corresponding intervals of the ir truth probabilities.

Let $\mathcal{B}$ be a such knowledge base, and $S$ be a goal sentence. The interval of truth probabilitics of $S$ derived from $\mathcal{B}$ can be found by the proposed method. 